\title{
Sachgerechtigkeit und Transparenz gehen vor Vereinfachung
}

\section{Urs Stoffel}

Dr. med., Mitglied des FMH-Zentralvorstandes, Departementsverantwortlicher Ambulante Versorgung und Tarife

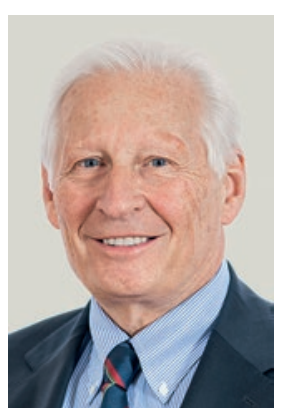

Von verschiedener Seite wird immer wieder die Vereinfachung des Einzelleistungstarifs TARDOC gefordert und im gleichen Atemzug werden die Vorteile von ambulanten Pauschalen hervorgehoben. Die Notwendigkeit eines Einzelleistungstarifs als Fundament und Basis aller ärztlichen Leistungen in der ambulanten Versorgung ist inzwischen jedoch unbestritten. Die gesetzliche Grundlage dazu fordert in Art. 43, Abs. 4 KVG, dass ein Einzelleistungstarif sachgerecht und betriebswirtschaftlich bemessen sein muss. Damit greift ein reiner Zeittarif zu kurz, um die gesetzlich geforderte Sachgerechtigkeit zu gewährleisten.

Nach diesen Kriterien und Vorgaben wurde der TARDOC in den letzten Jahren von den Tarifpartnern FMH, curafutura und MTK von Grund auf neu entwickelt und verhandelt. Die direkte Vergleichbarkeit einzelner Tarifpositionen von TARMED mit den neugeschaffenen Tarifpositionen im TARDOC ist daher nicht mehr gegeben.

Im Moment sind die Tarifpartner mit der Überprüfung der TARDOC-Struktur gemäss dem Prüfbericht des BAG beschäftigt. Die überarbeitete Tarifstruktur muss bis Ende Jahr von allen Tarifpartnern erneut eingereicht werden. FMH, curafutura und MTK haben den TARDOC hinsichtlich Vereinfachungspotenzial analysiert. Es lässt sich feststellen, dass eine Vereinfachung in vielen Fällen zulasten der Sachgerechtigkeit und der

Der Detaillierungsgrad der TARDOC-Tarifstruktur ermöglicht eine sachgerechte und transparente Abrechnung.

Transparenz gehen würde. Die Sachgerechtigkeit und Wirtschaftlichkeit haben für die Tarifpartner eine ebenso hohe Priorität wie ein vereinfachter Tarif. Der Detaillierungsgrad der Tarifstruktur bietet den Vorteil, dass die Anwendungs- und Abrechnungsregeln sowie die qualitativen Dignitäten klar definiert werden können. Auch die Minutagen der Handlungsleistungen können so präziser erfasst werden. Diese Vorteile dürfen nicht zugunsten einer unzweckmässigen Vereinfachung aufgegeben werden. Im Bereich der OP-Leistungen wird eine gewisse Vereinfachung aber erreicht werden können.

Zur Stärkung der Grundversorgung wurde für Hausärztinnen und Hausärzte im TARDOC ein eigenes Kapitel erstellt, das die Forderungen von Art. 117a BV erfüllt.

Der Grundsatz muss gelten: So einfach wie möglich und so differenziert wie nötig.

Beispielsweise fällt die Umfeldarbeit im hausärztlichen Bereich, wie die Betreuung von betagten Menschen, bei der Angehörige und Spitex involviert sind, umfangreicher aus als bei anderen Fachärzten. Diesem Umstand wird im Hausarztkapitel differenziert und transparent Rechnung getragen. Das Gleiche gilt für die Palliative Care.

Die Kumulation von Handlungs- und Zeitleistungen ist im TARDOC ein zentrales Tarifierungsinstrument. Dabei wurde darauf geachtet, dass die Inhalte von Grundleistung und Zuschlagsleistung klar definiert sind. Zeigen sich in der Anwendung tatsächlich kritische Abrechnungssituationen, werden diese im Rahmen des Monitorings zielgerichtet eruiert und korrigiert. Im Zeitalter der Digitalisierung, mit zunehmend informatisierten Praxisabrechnungen und der elektronischen Rechnungskontrolle bei den Versicherern, darf eine Vereinfachung des Tarifs auf Kosten der Transparenz und Sachgerechtigkeit nicht priorisiert werden. Die durchschnittliche Rechnung des Arztes in freier Praxis liegt unter CHF 300, umfasst etwas mehr als eine Sitzung und ist somit für die meisten Patientinnen und Patienten leicht nachzuvollziehen.

Der Grundsatz muss gelten: So einfach wie möglich und so differenziert wie nötig - genau das setzt der TARDOC konsequent um. 